
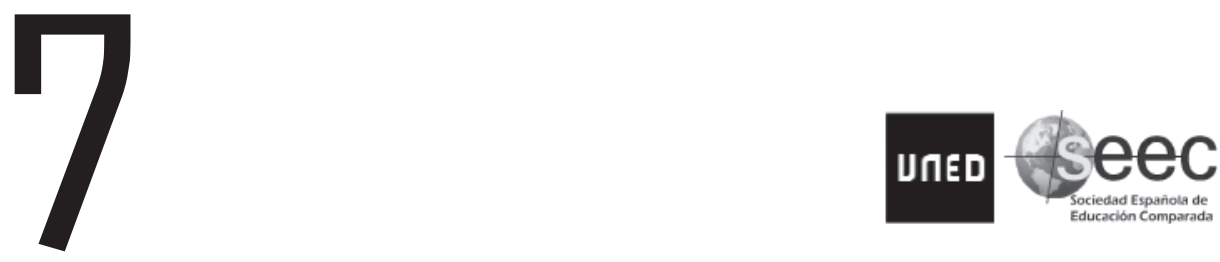

\title{
INTERVENCIONES DIRIGIDAS A LA PREVENCIÓN DEL FRACASO Y ABANDONO ESCOLAR: UN ESTUDIO DE REVISIÓN
}

\section{Interventions aimed at the prevention of failure and dropout at school: a review study}

\author{
Ma Isabel Miñaca * $^{*}$ \\ Mirian Hervás *
}

\section{RESUMEN}

En el presente estudio se revisan algunos programas de intervención tanto a nivel internacional como nacional relacionados con la prevención del fracaso y/o abandono escolar en la Educación Secundaria Obligatoria (ESO). Para ello, se realizó una búsqueda en las bases de datos ProQuest, ISOC, Redalyc y Dialnet sobre intervenciones realizadas desde el año 2000 al 2010 con dicho objetivo. Aunque entre ellas existen variaciones respecto a los objetivos, duración, actividades y resultados, todas ellas muestran unas características comunes a la hora de elaborar cualquier programa de esta tipología, que son convenientes tener en cuenta para la elaboración de

* Universidad de Huelva. 
actuaciones futuras que permitan una mejora del rendimiento académico del alumnado y un incremento de la promoción a niveles postobligatorios.

PALABRAS CLAVE: Fracaso escolar, Intervención escolar, Absentismo escolar, Educación secundaria obligatoria.

\begin{abstract}
In this study are reviewed some intervention programs both internationally and nationally related to the prevention of failure and/or school drop-outs in middle school. For this aim, carried out a search in the databases ProQuest, ISOC, Redalyc and Dialnet on interventions since 2000 to 2010 with the goal. Although between them variations exist as to the objectives, duration, activities and results, all of them show characteristics common to the time to develop any program of this type, which are suitable take into account for the development of future actions that would allow an improvement in the academic performance of the students and an increase in the promotion to high school.
\end{abstract}

KEY WORDS: School failure, Intervention school, Truancy, Middle school.

$* * * * *$

\title{
INTRODUCCIÓN
}

Las políticas educativas europeas (COMISIÓN DE LAS COMUNIDADES EUROPEAS, 2007) han mostrado a lo largo del último cuarto de siglo datos considerables sobre fracaso escolar y abandono del periodo educativo obligatorio y postobligatorio, que pueden ser factores de riesgo, exclusión social y pobreza, afectando a la población y evolución académica y profesional de los jóvenes afligidos y su consiguiente participación en la comunidad (EUROCHILD AISBL, 2007). Es por ello que el fracaso escolar es, en la actualidad, objeto de discusión, aún si sin establecerse una definición clara del mismo. A nivel español, se encuentran, por un lado, los que consideran que éste se refiere a no terminar la ESO; para otros, significa no superar la Educación Secundaria Postobligatoria, incluyendo suspensos, repeticiones de curso o el retraso educativo, y, un último grupo, va más allá, teniendo en cuenta las consecuencias del mismo en el alumno: 
descalificación y estigmatización del alumno, culpabilización y desresponsabilización (FERNÁNDEZ, MENA y RIVIERE, 2010).

En un intento de integración, el término fracaso escolar podría definirse como la situación del alumno que intenta alcanzar los objetivos mínimos educativos, es decir, los referidos a la ESO, pero no lo consigue y se retira (FERNÁNDEZ et al., 2010), mientras que el abandono escolar, incluiría a todos los alumnos entre 18 y 24 años que no han completado la Educación Secundaria Postobligatoria (Bachillerato y Ciclos Formativos de Grado Medio), incluyendo los que, aún finalizando la ESO (con o sin el título de graduado), no se han matriculado en ninguna de las modalidades del período educativo postobligatorio. Siguiendo esta línea, el fracaso estaría ligado a un posterior riesgo de abandono y viceversa, por lo que no se puede llegar a sostener que el fracaso y/o abandono escolar sean problemas insignificantes en nuestro sistema educativo, representado las dos caras de una misma moneda (GONZÁLEZ, 2006).

Aunque en la actualidad, el fracaso escolar se centra en la ESO, existen inicios en la educación primaria. Según los datos del informe «Fracaso y abandono escolar en España» de FERNÁNDEZ, et al. (2010), la repetición de curso comienza en la educación primaria: el 5,5\% de alumnos de 8 años y el 10\% de alumnos de 10 años, no asisten a los cursos que le corresponden por edad. A los 12 años (edad en la que se finaliza la etapa de educación primaria), existe un $15 \%$ de alumnos que no la finalizan y acumulan retraso. Estos datos sufren un incremento a medida que se avanza en el período educativo. Por ejemplo, a la edad de 16 años, un 33,8\% de los alumnos no han finalizado la educación secundaria, el 28,4\% de los alumnos abandonan sin obtener el título de graduado y el 14,8\% de ellos son de jóvenes menores de 16 años de edad. El $42 \%$ de los alumnos llega a $4^{\circ}$ de la ESO con retraso, y el $27 \%$ de ellos fue durante esta etapa. Además, se encuentran diferencias en cuanto al género, siendo los chicos los que presentan las tasas más elevadas: $35 \%$ de los chicos finaliza sin la obtención del graduado versus al 20\% de las chicas y, a la edad de 15 años, el 50\% de los chicos acumula fracaso, en comparación del $36 \%$ de las chicas.

Algunos de los indicadores previos del abandono escolar son la repetición y falta de asistencia al curso. Según los datos del informe del Consejo Económico y Social (2009), el 88\% de los alumnos que abandonan habían repetido curso alguna vez y el $91 \%$ presentaban faltas de asistencia no 
justificadas durante el curso académico. Cuando dichas faltas se suceden de forma reiterada y se extienden durante periodos de tiempo prolongados, el ritmo de aprendizaje de estos alumnos se ve afectado, comenzando a aparecer indicios de un posible retraso escolar, que, de no solucionarse a tiempo, originaría el posterior abandono (Sáez, 2005). En un estudio en el que participaron 7.168 estudiantes escolarizados en 83 centros educativos de Educación Secundaria de la Comunidad de Madrid (MARTÍNEZ-ARIAS et al., 2007) muestra que el $16,1 \%$ falta al colegio y un $24,1 \%$ a algunas de las clases un día o más en dos semanas. Por otro lado, un estudio canadiense de seguimiento de alumnos participantes en el Program for International Student Assessment (PISA) 2000, conocido con el nombre «Canadian Youth in Transitions Survey», mostró que los estudiantes con puntuaciones inferiores a las correspondientes al nivel 2 se enfrentan a un riesgo desproporcionalmente elevado de no continuar sus estudios en educación superior o de participar en desventaja en el mercado laboral a los 19 años, e incluso con perspectivas más desfavorables a los 21 años, la edad alcanzada por la cohorte estudiada mostrados en el último informe PISA del año 2009 (MINISTERIO DE EDUCACIÓN, 2009).

Este fracaso y posterior absentismo y/o abandono escolar, es un problema en el que juegan un papel activo tanto los factores educativos, personales y sociales. Las características sociodemográficas de las familias de estos jóvenes en riesgo de fracaso escolar y/o abandono son entre otras, la ocupación y nivel educativo: casi la mitad de los jóvenes que provienen de clases trabajadoras presenta un riesgo bastante elevado, frente a un $23 \%$ de las clases medias; más de la mitad de los alumnos con padres sin estudios tienen riesgo de fracaso escolar y casi la mitad de alumnos con padres que sólo cursaron estudios primarios (FERNÁNDEZ et al., 2010), capacidad para transmitir interés por los estudios y prestar ayuda y apoyo en las tareas escolares, el nivel socioeconómico, que suele ser medio-bajo y las expectativas y actitudes de las familias hacia el periodo educativo, expectativas académicas, implicación con el centro, condiciones de estudio en casa y relaciones familiares (RISSO, PERALBO y BARCA, 2010). Por ello, tanto el nivel educativo como el económico, cultural y social de las familias son variables relacionadas con el fracaso y posterior abandono escolar de los jóvenes tal y como revela el último informe PISA (2009) (MINISTERIO DE EDUCACIÓN, 2009). 
A todo lo citado se une, además, que la mayoría de los padres ejercen presión en dichos jóvenes para que sigan estudiando. Entre los principales motivos que atribuye dicho alumnado su abandono en la ESO, se encuentra el valor relativo de la escuela y la prioridad de la práctica que proporciona un trabajo, la influencia del grupo de iguales y la atracción que tiene un empleo para poder acceder y adquirir bienes materiales de consumo y una mayor independencia (CONSEJO ECONÓMICO y SOCIAL, 2009). Por otro lado, entre los factores personales asociados se encuentran la autoestima, la valoración positiva, autonomía y capacidad de autocontrol; aspectos cognitivos, como la estabilidad emocional, empatía, y la interacción con el grupo de iguales y habilidades sociales.

En esta línea, algunos autores, como Sáez (2005) están de acuerdo en que ha de tenerse en cuenta medidas tanto a nivel educativo como familiares para la elaboración de soluciones dirigidas a combatir esta problemática, puesto que entre las diferentes causas del fracaso y/o abandono escolar, se encuentran:

1. El entorno en el que vive el alumno, como pieza fundamental para el desarrollo de comportamientos y actitudes. La sensación de inutilidad que transmite la institución escolar, al no atender a las necesidades vitales de dichos jóvenes, el seguimiento personal y familiar insuficiente proporcionado y el desinterés que muestra este alumnado, origina que este entorno difícilmente pueda ser reforzante a la hora de prevenir este fracaso. A todo ello, se une que la escuela debe atender y adaptarse a las necesidades de cada colectivo y debe de satisfacer sus expectativas.

2. La institución escolar. Las medidas que desde la escuela se proponen para hacer frente a esta problemática suelen ser insuficientes, por lo que los índices de fracaso y/o posterior abandono escolar se mantienen y perduran a lo largo del tiempo. El alumno no termina de encontrar su sitio en el sistema educativo, lo que conlleva a distanciarse y terminar rompiendo con el sistema educativo.

3. La situación familiar. La precariedad económica, nivel educativo, relaciones familiares, expectativas, importancia otorgada a la educación, etc., son entre otros, algunos de los factores familiares asociados al fracaso escolar. Para la elaboración de soluciones 
posibles a esta problemática, se ha de tener en cuenta la creación de medidas en las que estas familias vieran cumplidas sus expectativas, produciéndose un acercamiento entre los agentes educativos y familiares.

4. El grupo de iguales. Este grupo, ejerce una influencia en la toma de decisiones, normas, intereses y necesidades del sujeto.

5. El propio alumno/a. El desinterés que manifiestan muchos jóvenes puede generar, en la mayoría de los casos, una baja autoestima y falta de competencias, lo que podría generar un fracaso y posterior absentismo escolar.

Dentro de las políticas preventivas para el tratamiento del fracaso escolar se proponen entre otras (CONSEJO ECONÓMICO y SOCIAL, 2009; NAVARRETE, 2007): (a) proporcionar desde las Administraciones recursos humanos y materiales para dar apoyo a estos jóvenes durante el horario extraescolar; (b) informar y concienciar a la sociedad a través de campañas divulgativas centradas en esta problemática; (c) elaborar informes para tener un mejor conocimiento de las causas que están detrás de todo ello y de su incidencia entre las mujeres y hombres; (d) aumentar las expectativas y estrategias individuales y familiares en relación con la educación; (e) promover investigaciones que se centren en territorios y colectivos específicos; (f) reforzar la orientación académica y profesional de los centros educativos; (g) desarrollar itinerarios de formación personalizados y adaptados a cada sujeto; (h) potenciar la autonomía organizativa y de gestión de los centros escolares para que puedan atender de manera específica al alumnado con estas características y que cuenten con el respaldo de la Administración Educativa; (i) fomentar la participación de los padres en los centros educativos e incrementar la coordinación escuela-familia; y (j) avivar la comunicación entre las Comunidades Educativas, para conocer los resultados de otros centros, localidades y provincias, prácticas docentes, sistemas, mecanismos, prácticas reales de evaluación y promoción. Por otro lado, dentro de las áreas de cooperación entre la escuela y la familia para poder atender e intervenir de una manera más efectiva con este colectivo se encuentra (MARTÍNEZ y ÁLVAREZ, 2005): (1) realizar tutorías para padres/madres/tutores, para poder informar sobre los recursos educativos del centro; (2) crear escuelas de padres; (3) proporcionar información al centro sobre el desarrollo físico, afectivo, social y/o motivaciones de los jóvenes por 
parte de las familias; (4) implicar de las familias en las actividades de aprendizaje de sus hijos; (5) consultar a menudo la trayectoria educativa de sus hijos; (6) proporcionar a dichas familias orientación sobre hábitos de estudio; (7) fomentar la participación de los padres/madres/tutores en los órganos de gestión del centro (Ej., Asociación de Madres y Padres -AMPAs-, Consejo escolar, etc.); y (8) promover la comunicación del centro y familias con otras entidades comunitarias para complementar la oferta educativa en materias transversales.

Es por ello que el reto para abordar esta problemática es la coordinación de los diferentes agentes implicados y no por separado. En un intento de prevenir este fracaso y/o abandono escolar durante el periodo educativo obligatorio, se han elaborado diferentes propuestas tanto a nivel nacional -Ej., Programas de Cualificación Profesional Inicial (PCPI) del Ministerio de Educación y Ciencia (Ley Orgánica de Educación, 2006) - como internacional (MCCLUSKEY, BYNUM y PATCHIN, 2004). En esta línea el presente trabajo tiene como objetivo realizar una revisión de los resultados de los programas de intervención relacionados con el fracaso y/o abandono escolar, tanto a nivel nacional como internacional, para poder recopilar los aspectos o ámbitos necesarios que han de tenerse en cuenta en este tipo de intervenciones y tenerlas presentes en futuras medidas a elaborar dirigidas a esta problemática, así como ofrecer información a los profesionales de las diferentes propuestas llevadas a cabo para intervenciones futuras.

\section{MÉTODO}

Para llevar a cabo el objetivo propuesto, se ha realizado una revisión de la literatura entre los años 2002 y 2010. Se realizó una búsqueda en las bases de datos: ProQuest, ISOC, Redalyc y Dialnet. En cuanto a los descriptores, se incluyeron las diferentes palabras clave: «intervention school failure», «truancy», «fracaso escolar en secundaria», «intervención escolar», «absentismo escolar», «absenteeism», «prevención escolar», «truancy interventions», «prevención absentismo escolar», «prevención fracaso escolar», «intervención fracaso escolar», «school failure» e «intervención absentismo escolar», entre las más importantes. 
De un total de 15 trabajos encontrados, se descartaron, por un lado, 3, al atender a población universitaria de nuevo ingreso y al no presentar datos sobre la evaluación del mismo, respectivamente; y, por otro, otras 3 por ser tesis doctorales, 2 de ellas americanas y 1 inglesa. En total se seleccionaron nueve artículos referidos a programas de intervención para la prevención del fracaso y absentismo escolar, cinco de ellos realizados en España y cuatro de ellos llevados a cado en Estados Unidos (EEUU). Estos programas de intervención, fueron seleccionados atendiendo a una serie de criterios: (1) programas dirigidos a colectivos de la ESO o nivel similar; (2) presentar información sobre los objetivos propuestos, duración del mismo y actividades a realizar; y (3) presentar datos de resultados tras su aplicación, que permitan la evaluación del programa y poder analizar la eficacia o no del mismo para poder ser comparados entre sí.

\section{RESULTADOS}

A continuación, aparece un resumen de los programas de intervención encontrados que son presentados en la Tabla 1.

Se han ordenado alfabéticamente identificando el trabajo (autores y año de publicación), nombre del programa, lugar de realización, colectivo al que va dirigido, objetivos del programa, duración de la intervención, actividades y resultados obtenidos tras la aplicación. 


\section{Tabla 1. Resumen de los programas de intervención dirigidos} a la prevención del fracaso y abandono escolar

\begin{tabular}{|c|c|c|c|c|c|c|}
\hline $\begin{array}{c}\text { Autores } \\
\text { y año }\end{array}$ & $\begin{array}{c}\text { Nombre del } \\
\text { programa }\end{array}$ & $\begin{array}{l}\text { Lugar de real- } \\
\text { ización }\end{array}$ & Colectivo & Objetivos & $\begin{array}{l}\text { Duración / } \\
\text { Actividades }\end{array}$ & Resultados \\
\hline 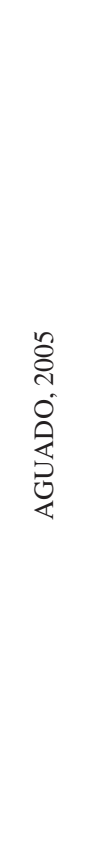 & $\begin{array}{l}\text { Prevención } \\
\text { y control } \\
\text { del absen- } \\
\text { tismo esco- } \\
\text { lar. }\end{array}$ & Madrid. & $\begin{array}{l}\text { Alumnos ab- } \\
\text { sentistas de } \\
21 \text { distritos } \\
\text { de la Comu- } \\
\text { nidad de Ma- } \\
\text { drid. }\end{array}$ & $\begin{array}{l}\text { Procurar una } \\
\text { asistencia regu- } \\
\text { lar a clase, in- } \\
\text { crementar el } \\
\text { contacto con la } \\
\text { familia y las } \\
\text { situaciones fa- } \\
\text { miliares que } \\
\text { pueden provo- } \\
\text { car absentismo, } \\
\text { fomentar la par- } \\
\text { ticipación de } \\
\text { los jóvenes en } \\
\text { actividades so- } \\
\text { cioeducativas } \\
\text { en Organizacio- } \\
\text { nes no Guber- } \\
\text { namentales } \\
\text { (ONGs), coor- } \\
\text { dinación de ent- } \\
\text { idades para es- } \\
\text { tablecer } \\
\text { criterios de in- } \\
\text { tervención ori- } \\
\text { entados a la } \\
\text { prevención del } \\
\text { absentismo. }\end{array}$ & $\begin{array}{l}\text { Desde el } 2001 \\
\text { hasta la actuali- } \\
\text { dad/ Conocimien- } \\
\text { to y trámites para } \\
\text { identificación de } \\
\text { casos, Interven- } \\
\text { ción familiar, co- } \\
\text { munitaria e indi- } \\
\text { vidual, campañas } \\
\text { de información, } \\
\text { promoción de ac- } \\
\text { ceso a la edu- } \\
\text { cación infantil, } \\
\text { programa de } \\
\text { acogida, programa } \\
\text { para la utilización } \\
\text { del tiempo libre, } \\
\text { seguimiento de la } \\
\text { escolarización, ac- } \\
\text { ción tutorial y de } \\
\text { orientación educa- } \\
\text { tiva. }\end{array}$ & $\begin{array}{l}\text { Incremento de } \\
\text { la participación } \\
\text { e implicación de } \\
\text { los centros edu- } \\
\text { cativos, aumen- } \\
\text { to de la sensibil- } \\
\text { idad de los } \\
\text { sectores de la } \\
\text { Comunidad y de } \\
\text { las familias. }\end{array}$ \\
\hline 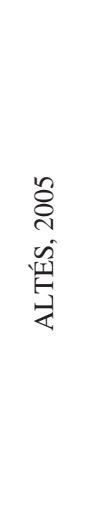 & Aula 15. & $\begin{array}{l}\text { Vila Seca, } \\
\text { Tarragona. }\end{array}$ & $\begin{array}{l}\text { Los dos In- } \\
\text { stitutos de } \\
\text { Educación } \\
\text { Secundaria } \\
\text { (IES) del } \\
\text { municipio. }\end{array}$ & $\begin{array}{l}\text { Proporcionar } \\
\text { atención ped- } \\
\text { agógica a alum- } \\
\text { nos que no se } \\
\text { adaptan al siste- } \\
\text { ma escolar, } \\
\text { para prevenir el } \\
\text { absentismo y } \\
\text { desescolar- } \\
\text { ización. }\end{array}$ & $\begin{array}{l}\text { Desde el curso } \\
\text { 2001/2002 hasta } \\
\text { la actualidad/ } \\
\text { Talleres preprofe- } \\
\text { sionalizantes (car- } \\
\text { pintería, cocina, } \\
\text { jardinería); Áreas } \\
\text { instrumentales; } \\
\text { Educación física, } \\
\text { visual y plástica; } \\
\text { Temas transver- } \\
\text { sales (educación } \\
\text { emocional, sexual, } \\
\text { etc.); orientación y } \\
\text { tutoría, grupal e } \\
\text { individual. }\end{array}$ & $\begin{array}{l}\text { El absentismo y } \\
\text { la desescolar- } \\
\text { ización ha dis- } \\
\text { minuido en un } \\
70 \% \text {. Incremen- } \\
\text { to de la partici- } \\
\text { pación e impli- } \\
\text { cación de la } \\
\text { familia. }\end{array}$ \\
\hline
\end{tabular}




\begin{tabular}{|c|c|c|c|c|c|c|}
\hline $\begin{array}{l}\text { Autores } \\
\text { y año }\end{array}$ & $\begin{array}{l}\text { Nombre del } \\
\text { programa }\end{array}$ & $\begin{array}{l}\text { Lugar de real- } \\
\text { ización }\end{array}$ & Colectivo & Objetivos & $\begin{array}{l}\text { Duración / } \\
\text { Actividades }\end{array}$ & Resultados \\
\hline $\begin{array}{l}0 \\
0 \\
0 \\
0 \\
0 \\
0 \\
0\end{array}$ & $\begin{array}{l}\text { Evaluación } \\
\text { del Pro- } \\
\text { grama Re- } \\
\text { fuerzo, Ori- } \\
\text { entación y } \\
\text { Apoyo } \\
\text { (PROA). }\end{array}$ & Zaragoza. & $\begin{array}{l}\text { IES «El Por- } \\
\text { tillo». } 45 \\
\text { alumnos de } \\
\text { primer ciclo } \\
\text { de la ESO } \\
\text { que presen- } \\
\text { tan algún re- } \\
\text { traso escolar, } \\
\text { bajas expec- } \\
\text { tativas aca- } \\
\text { démicas, es- } \\
\text { casa } \\
\text { participación } \\
\text { en activi- } \\
\text { dades, ausen- } \\
\text { cia de hábi- } \\
\text { tos de } \\
\text { trabajo. }\end{array}$ & \begin{tabular}{|l|} 
Realizar una \\
valoración de \\
este Programa \\
para analizar \\
los fallos en su \\
implementación \\
y que pueda \\
servir a modo \\
informativo \\
para profesion- \\
ales y mejorar \\
este tipo de in- \\
tervenciones en \\
los centros edu- \\
cativos.
\end{tabular} & $\begin{array}{l}\text { Curso 2006/2007, } \\
\text { ocho meses/ Tran- } \\
\text { sición de primaria } \\
\text { a secundaria, re- } \\
\text { fuerzo educativo, } \\
\text { biblioteca escolar, } \\
\text { colaboración con } \\
\text { familias, acompa- } \\
\text { ñamiento aca- } \\
\text { démico y activi- } \\
\text { dades } \\
\text { extraescolares. }\end{array}$ & $\begin{array}{l}\text { No se encuen- } \\
\text { tran diferencias } \\
\text { en relación a la } \\
\text { mejora del ren- } \\
\text { dimiento aca- } \\
\text { démico. Se re- } \\
\text { quiere mayor } \\
\text { implicación por } \\
\text { parte del profe- } \\
\text { sorado. }\end{array}$ \\
\hline 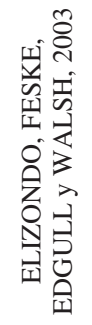 & $\begin{array}{l}\text { Programa } \\
\text { de Mejora } \\
\text { del Ab- } \\
\text { sentismo } \\
\text { Escolar. }\end{array}$ & California. & $\begin{array}{l}\text { Alumnos de } \\
\text { Primaria, se- } \\
\text { cundaria y } \\
\text { Educación } \\
\text { Secundaria } \\
\text { posobliga- } \\
\text { toria de Sali- } \\
\text { nas. }\end{array}$ & $\begin{array}{l}\text { Reducir la tasa } \\
\text { de absentismo } \\
\text { escolar. }\end{array}$ & $\begin{array}{l}\text { Curso escolar/ Ac- } \\
\text { tividades en las } \\
\text { que se implicaron } \\
\text { los agentes de la } \\
\text { comunidad, famil- } \\
\text { ias, expertos de la } \\
\text { salud mental y es- } \\
\text { tudiantes. }\end{array}$ & $\begin{array}{l}\text { Tras participar } \\
\text { en el programa, } \\
\text { los estudiantes } \\
\text { redujeron su } \\
\text { tasa de absentis- } \\
\text { mo en más de } \\
\text { un } 50 \%\end{array}$ \\
\hline 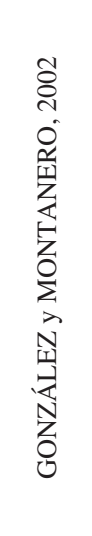 & $\begin{array}{l}\text { Acción tu- } \\
\text { torial. }\end{array}$ & Salamanca. & $\begin{array}{l}\text { IES privado. } \\
\text { Alumnos de } \\
3^{\circ} \text { de la ESO } \\
\text { con bajo ren- } \\
\text { dimiento es- } \\
\text { colar. }\end{array}$ & $\begin{array}{l}\text { Mejorar el ren- } \\
\text { dimiento esco- } \\
\text { lar en los par- } \\
\text { ticipantes del } \\
\text { programa, es } \\
\text { decir, incre- } \\
\text { mentar el } \\
\text { número de } \\
\text { aprobados. }\end{array}$ & $\begin{array}{l}3 \text { meses / control } \\
\text { estimular externo } \\
\text { e interno, reflex- } \\
\text { ión, compromiso } \\
\text { con metas, pro- } \\
\text { gramación activi- } \\
\text { dades de estudio, } \\
\text { auto-observación, } \\
\text { auto-refuerzo de } \\
\text { hábitos, interven- } \\
\text { ción con padres, } \\
\text { técnicas de con- } \\
\text { certación de com- } \\
\text { promisos, pro- } \\
\text { gramación } \\
\text { semanal y auto- } \\
\text { registro. }\end{array}$ & $\begin{array}{l}\text { Reducción de } \\
\text { las conductas } \\
\text { disruptivas. No } \\
\text { hubo diferencias } \\
\text { estadísticamente } \\
\text { significativas } \\
\text { entre el grupo } \\
\text { intervención y } \\
\text { control en la } \\
\text { media de asig- } \\
\text { naturas aproba- } \\
\text { das. }\end{array}$ \\
\hline
\end{tabular}




\begin{tabular}{|c|c|c|c|c|c|c|}
\hline $\begin{array}{l}\text { Autores } \\
\text { y año }\end{array}$ & $\begin{array}{c}\text { Nombre del } \\
\text { programa }\end{array}$ & $\begin{array}{l}\text { Lugar de real- } \\
\text { ización }\end{array}$ & Colectivo & Objetivos & $\begin{array}{l}\text { Duración / } \\
\text { Actividades }\end{array}$ & Resultados \\
\hline 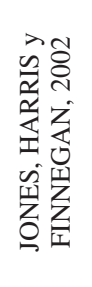 & $\begin{array}{l}\text { Programa } \\
\text { de Asisten- } \\
\text { cia Escolar. }\end{array}$ & San Diego. & $\begin{array}{l}\text { Estudiantes } \\
\text { entre } 16 \text { y } 18 \\
\text { años de San } \\
\text { Diego. }\end{array}$ & $\begin{array}{l}\text { Incrementar la } \\
\text { tasa de asisten- } \\
\text { cia a clase y la } \\
\text { graduación en } \\
\text { la ESO }\end{array}$ & $\begin{array}{l}19 \text { meses / Control } \\
\text { de asistencia a } \\
\text { clase, actividades } \\
\text { grupales, individu- } \\
\text { ales, familiares, } \\
\text { contratos conduc- } \\
\text { tuales de compro- } \\
\text { miso con el ado- } \\
\text { lescente. }\end{array}$ & $\begin{array}{l}\text { Incremento de } \\
\text { la asistencia a } \\
\text { clase a favor del } \\
\text { grupo interven- } \\
\text { ción, de un 3\% a } \\
\text { un } 9 \%\end{array}$ \\
\hline 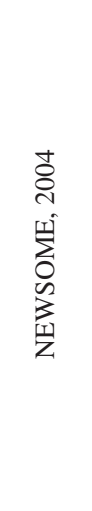 & $\begin{array}{l}\text { Interven- } \\
\text { ción con fa- } \\
\text { milias y es- } \\
\text { tudiantes } \\
\text { para la pre- } \\
\text { vención del } \\
\text { absentismo. }\end{array}$ & Ohio & $\begin{array}{l}13 \text { Estudi- } \\
\text { antes de } \\
\text { ESO. }\end{array}$ & $\begin{array}{l}\text { Reducir la tasa } \\
\text { de absentismo } \\
\text { escolar en los } \\
\text { participantes } \\
\text { tomando como } \\
\text { línea base el } \\
\text { número de } \\
\text { faltas de asis- } \\
\text { tencia en el cur- } \\
\text { so pasado. }\end{array}$ & $\begin{array}{l}8 \text { semanas del cur- } \\
\text { so escolar / Pre- } \\
\text { sentación, estab- } \\
\text { lecimiento de } \\
\text { metas a conseguir, } \\
\text { objetivos actuales } \\
\text { y metas futuras, } \\
\text { grupos de dis- } \\
\text { cusión sobre la } \\
\text { consecución de } \\
\text { objetivos propues- } \\
\text { tos y alcanzados, } \\
\text { refuerzo para el } \\
\text { cambio. }\end{array}$ & $\begin{array}{l}\text { No se encon- } \\
\text { traron diferen- } \\
\text { cias estadística- } \\
\text { mente } \\
\text { significativas } \\
\text { entre el grupo } \\
\text { intervención y } \\
\text { control. Si hubo } \\
\text { diferencias a fa- } \\
\text { vor del grupo } \\
\text { intervención en } \\
\text { la relación con } \\
\text { los iguales, au- } \\
\text { toestima y con- } \\
\text { trol de emocio- } \\
\text { nes. }\end{array}$ \\
\hline 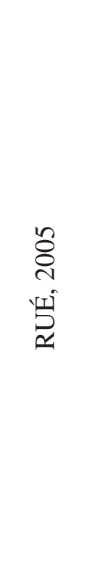 & $\begin{array}{l}\text { La mejora } \\
\text { del éxito } \\
\text { escolar. }\end{array}$ & $\begin{array}{l}\text { Martorell, } \\
\text { Calella, } \\
\text { Sabadell y } \\
\text { Vic. }\end{array}$ & $\begin{array}{l}\text { Estudiantes } \\
\text { de los IES de } \\
\text { Martorell, } \\
\text { Calella, Sab- } \\
\text { adell y Vic. }\end{array}$ & $\begin{array}{l}\text { Desarrollar pro- } \\
\text { puestas de tra- } \\
\text { bajo operativas } \\
\text { para los alum- } \\
\text { nos en riesgo } \\
\text { social que ga- } \\
\text { rantice una me- } \\
\text { jora del éxito } \\
\text { escolar. }\end{array}$ & $\begin{array}{l}\text { Curso } 2003 \text { a la } \\
\text { actualidad/ Taller } \\
\text { de comunicación, } \\
\text { científica tec- } \\
\text { nológicas, } \\
\text { matemáticas, vida } \\
\text { social, salud, hi- } \\
\text { giene y preven- } \\
\text { ción, relaciones } \\
\text { interpersonales, } \\
\text { estética, artes y } \\
\text { oficios, Medio } \\
\text { ambiente, razona- } \\
\text { miento moral, } \\
\text { comprensión so- } \\
\text { ciohistórica, rela- } \\
\text { ciones económi- } \\
\text { cas. }\end{array}$ & $\begin{array}{l}\text { Mejora en el } \\
\text { éxito escolar en } \\
\text { el curso } 2003 . \\
\text { No se disponen } \\
\text { datos de los } \\
\text { demás años. }\end{array}$ \\
\hline 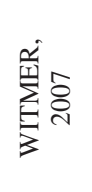 & $\begin{array}{l}\text { Programa } \\
\text { para la me- } \\
\text { jora del ab- } \\
\text { sentismo } \\
\text { escolar. }\end{array}$ & $\begin{array}{l}\text { Pennsylva- } \\
\text { nia. }\end{array}$ & $\begin{array}{l}73 \text { jóvenes } \\
\text { afroamerica- } \\
\text { nos. }\end{array}$ & $\begin{array}{l}\text { Reducir la tasa } \\
\text { de absentismo } \\
\text { escolar y las } \\
\text { conductas dis- } \\
\text { ruptivas. }\end{array}$ & $\begin{array}{l}10 \text { meses / Refuer- } \\
\text { zo académico, } \\
\text { plan de estudio, } \\
\text { habilidades socia- } \\
\text { les, orientación } \\
\text { laboral. }\end{array}$ & $\begin{array}{l}\text { Incremento de } \\
\text { la tasa de asis- } \\
\text { tencia a clase y } \\
\text { reducción de } \\
\text { conductas dis- } \\
\text { ruptivas. }\end{array}$ \\
\hline
\end{tabular}




\subsection{Características de los programas}

Aunque cada programa se plantea conseguir unos objetivos diferentes, todos ellos parten del mismo propósito: reducir la tasa de absentismo escolar, traducido en un incremento de la asistencia a clase por parte del alumno y mejorar el rendimiento escolar. Todos los programas plantean un marco teórico previo en el que se explica de forma clara y precisa la problemática del fracaso en la actualidad, y la necesidad de elaborar y crear por parte de los profesionales propuestas para poder hacerle frente y reducir las altas tasas de fracaso y abandono existentes, tanto a nivel nacional como internacional.

Respecto al contenido y desarrollo de las intervenciones, observando la Tabla 1 se puede concluir que no siempre se especifican y aclaran. Aunque las actividades planteadas en los programas son muy diversas, se puede observar que las dedicadas al refuerzo académico, acción tutorial, intervención familiar, habilidades sociales y técnicas de estudio son las más comunes. Además, se puede observar que la mayoría de estos programas de intervención tienen una duración media de 10-12 meses, es decir, el curso académico. Solamente dos de las intervenciones presentadas en la Tabla 1 presentan una duración inferior a la media (GONZÁLEZ y MONTANERO, 2002; NEWSOME, 2004).

Todos los programas aquí presentados, están elaborados para llevarse a cabo con alumnos pertenecientes a la ESO, con una edad comprendida entre 13-16 años en su mayoría, exceptuando a tres de estos programas (BROC, 2010; GONZÁLEZ y MONTANERO, 2002; NEWSOME, 2004), que están dirigidos a una serie de alumnos con unas características determinadas, como es que presenten bajo rendimiento escolar y retraso escolar y uno de ellos (JONES et al., 2002), que está dirigido a alumnos de entre 16 y 18 años y otro (ELIZONDO et al., 2003) se llevó a cabo tanto con alumnos pertenecientes a la Educación Primaria, E.S.O. y Educación Secundaria Postobligatoria.

Por último, se puede observar que la mayoría de estas intervenciones presentan resultados favorables tras su finalización, incrementando la tasa de asistencia a clase, reducción de conductas disruptivas, incremento del número de asignaturas aprobadas e implicación de la familia y la comunidad, exceptuando uno (BROC, 2010) en el que no se encontraron diferencias tras la aplicación del mismo debido a la falta de implicación del profesorado e inicio tardío en la aplicación del mismo por problemas burocráticos y administrativos. 


\section{DISCUSIÓN}

Analizando los resultados obtenidos presentados en la Tabla 1, se puede establecer a simple vista, las características comunes entre los programas presentados para tenerlas en cuenta a la hora de elaborar intervenciones futuras.

En primer lugar, se observa que todos los programas aquí expuestos, presentan unos resultados tras su aplicación bastante favorables en su mayoría, por lo que cualquier mínima intervención dedicada a prevenir el fracaso y/o abandono escolar puede ser bienvenida. Además, no solo pueden conseguir el efecto directo de un incremento de asistencia a clase o mejora del rendimiento académico, sino que indirectamente, también producen otros efectos secundarios, tales como la mejora de las relaciones con los iguales, disminución de conductas disruptivas, mejora del comportamiento en el aula, control de emociones e incremento de la motivación y autoestima (GONZÁLEZ y MONTANERO, 2002; NEWSOME, 2004; WITMER, 2007).

En segundo lugar, se puede observar que la mayoría de ellos, incluyen a todos los agentes implicados en el fracaso y/o abandono escolar de los jóvenes (familia, comunidad y profesorado) a través de la realización de actividades, obteniendo en los resultados tras la aplicación una mayor implicación y compromiso de los mismos, por lo que se podría establecer que uno de los puntos relevantes a la hora de elaborar posibles programas de intervención para la prevención de esta problemática, es la de llevar a cabo acciones que comprometan a todos los agentes implicados, tal y como señala la bibliografía especializada (CONSEJO ECONÓMICO Y SOCIAL, 2009; FERNÁNDEZ et al., 2010; NAVARRETE, 2007).

En tercer lugar, puede observarse que las actividades a realizar con los alumnos más comunes entre los programas aquí presentados están relacionadas con actividades dedicadas al refuerzo académico, planificación y organización del tiempo y acción tutorial, junto con contratos conductuales en los que dichos jóvenes manifiesten su implicación y compromiso con el programa (AGUADO, 2005; BROC, 2010; GONZÁLEZ y MONTANERO, 2002; JONES et al., 2002; NEWSOME, 2004; WITMER, 2007), por lo que éstas podrían ser las actividades básicas a incluir en cualquier programa dedicado a la prevención e intervención del fracaso o absentismo escolar.

En cuarto lugar, se observa que en la mayor parte de ellos, la duración de los programas es prácticamente igual, por lo que éste es otro de los aspectos 
a tener en cuenta a la hora de elaborar cualquier programa de estas características. Puesto que el objetivo es reducir la tasa de fracaso escolar y/o abandono, lo recomendable es que dicha intervención aborde todo el curso académico y que las actividades a llevar a cabo sean semanales, tal y como muestran los resultados obtenidos de dichos programas de intervención.

\section{CONCLUSIÓN}

Según datos extraídos del Informe PISA 2009 (MINISTERIO DE EDUCACIÓN, 2009) seguimos muy por debajo de la media europea respecto a las competencias básicas, por lo que, dichos resultados, reflejan la necesidad de crear intervenciones y de establecer medidas para la mejora del rendimiento y prevención del absentismo y abandono académico del sistema educativo. Dos de los objetivos principales que se tiene que plantear cualquier tipo de intervención con estas características es, por un lado, la de que los alumnos consigan mejorar su rendimiento académico y promocionen al siguiente curso, disminuyendo por ende las tasas de fracaso y abandono escolar y, por otro, concretarse en el desarrollo de programas que incluyan un sistema de registro y comunicación institucional entre todos los agentes, que hagan posible detectar la situación en la que se encuentra cada alumno y, al mismo tiempo, la elaboración de estudios para la creación de proyectos de intervención educativa con los agentes implicados (SÁEZ, 2005).

En esta línea, si bien los datos de fracaso y absentismo escolar (en torno al 30\%) de los últimos informes a nivel nacional (CONSEJO ECONÓMICO Y SOCIAL, 2009; FERNÁNDEZ et al., 2010), junto con los extraídos del último informe PISA (2009) (MINISTERIO DE EDUCACIÓN, 2009) son bastantes preocupantes, bien es cierto también que las diferentes alternativas creados para combatirlos están dando resultado. Por ejemplo, entre los diferentes programas mostrados en la Tabla 1, se puede observar reducciones de hasta el $70 \%$ en las tasas de absentismo y desescolarización de los participantes (ALTÉS, 2005; ELIZONDO et al., 2003) e incremento de asistencia a clase de un 3\% a un 9\% (JONES et al., 2002), lo que originaría un posterior incremento en la tasa de graduación y empleabilidad y por consiguiente, disminuiría el riesgo de exclusión y pobreza (EUROCHILD, AISLB, 2007). Además, la eficacia de este tipo de programas, no solo han de traducirse en disminución del absentismo e incremento de asistencia a clase, sino que también muestran resultados favorables al control de emociones y disminución de conductas 
disruptivas (GONZÁLEZ y MONTANERO, 2002; NEWSOME, 2004; WITMER, 2007) e incremento en la participación e implicación de los agentes implicados en esta problemática (Ej., familias, personal docente, etc.) (AGUADO, 2005; ALTÉS, 2005), ingrediente necesario según los expertos para que la eficiencia y eficacia de dichas alternativas (CONSEJO ECONÓMICO y SOCIAL, 2009; NAVARRETE, 2007).

En definitiva, este trabajo pretende dar a conocer diferentes iniciativas puestas en práctica, las cuales no han estado exentas de limitaciones y problemáticas en su desarrollo, teniendo como finalidad mejorar gran parte de las necesidades y desafíos que conlleva el proceso de Enseñanza-Aprendizaje (E-A), que actualmente se plantean a profesorado, alumnado y responsables institucionales. Para ello, se necesitará disponer de mecanismos de detección precoz del evidente fracaso académico y escolar, así como medidas de ayuda para contrarrestar los altos niveles del mismo, que permitan aumentar los índices de graduación, además de una mayor corresponsabilidad entre las partes. En todo caso, y mediante estas experiencias, el alumnado participante ha mejorado su productividad, ha incidido en factores cognitivos, emocionales y comportamentales en el desarrollo de las capacidades de autorregulación, así como la adquisición de competencias, habilidades, destrezas, conocimientos, valores etc., adaptados a su situación específica, con los que llegar a convertirse en unos ciudadanos cívicos.

\section{REFERENCIAS BIBLIOGRÁFICAS}

AGUADO GONZÁlEZ, P. (2005): Programa de Prevención y Control del Absentismo Escolar en el Ayuntamiento de Madrid. INDIVISA, Boletín de Estudios e Investigación, 6, pp. 241-249.

ALTÉS, J. M. y DOMÍNGUEZ, M. J. (2005): Aula 15: el abordaje del absentismo en la ESO, Revista de Educación Social, 4 (http://www. eduso.net $/ \mathrm{res} / \mathrm{l} \mathrm{b}=7 \& \mathrm{c}=53 \& \mathrm{n}=160)$.

BROC, M. A. (2010): Estudio investigación valorativa de la eficacia del Programa de Refuerzo, Orientación y Apoyo (PROA) en alumnos de Educación Secundaria Obligatoria, Revista de Educación, 352, pp. 405-429.

COMISIÓN DE LAS COMUNIDADES EUROPEAS (2007): Fomentar la plena participación de los jóvenes en la educación, el empleo y la sociedad. COM (2007) 498 final. 
CONSEJO ECONÓMICO Y SOCIAL (2009): Sistema educativo y capital humano en España (Madrid, UGT-FETE Enseñanza).

ELIZONDO, F., FESKE, K., EDGULL, D. y WALSH, K. (2003): Creating synergy through collaboration: Safe schools/healthy students in Salinas, California, Psychology in the Schools, 40, pp. 503-513.

EUROCHILD AISBL (2007): Ending Child Poverty within the EU? A review of the 2006-08 national reports on strategies for social protection and social inclusion ( $2^{\text {nd }}$ Edition) (http://www.icyrnet.net/UserFiles/File/ NAPs\%20report\%202006\%20final.pdf).

FERNÁNDEZ ENGUITA, M., MENA, L. y RIVIERE, J. (2010): Fracaso y abandono escolar en España (Barcelona, Colección de Estudios Sociales «La Caixa»).

GONZÁLEZ GONZÁLEZ, M. T. (2006): Absentismo y abandono escolar: una situación singular de la exclusión educativa, Revista electrónica Iberoamericana sobre Calidad, Eficacia y Cambio en Educación, 4/1, pp. 1-15.

GONZÁlEZ, L. y MONTANERO, M. (2002): Cómo mejorar la autorregulación del estudio en la educación secundaria. Valoración de in programa de acción tutorial, Contextos educativos, 5, pp. 215-226.

JONES, L. P., HARRIS, R. y FINNEGAN, D. (2002): School attendance demonstration project: An evaluation of a program to motivate public assistance teens to attend and complete school in an urban school district, Research and Social Work Practice, 12, pp. 222-237.

LEY ORGÁNICA DE EDUCACIÓN (2006). Ley Orgánica 2/2006, de 3 de mayo, de Educación (Publicada en BOE n. ${ }^{\circ}$ 106, de 4 de mayo de 2006). Extraído el 20 de Febrero de 2012, de http://todofp.es/dctm/todofp/ legislacion/a17158-17207.pdf?documentId=0901e72b800d0145

MARTÍNEZ-ARIAS, R., AGUADO, P., ÁLVAREZ-MONTESERÍN, A., COLODRÓN, F. y GALLEGO, E. (2007): Prevalencia del absentismo escolar en educación secundaria obligatoria: relaciones con variables sociodemográficas y familiares, Revista de Psicología Educativa, 13/1, pp. 35-63.

MARTÍNEZ GONZÁLEZ, R. y ÁLVAREZ BLANCO, L. (2005): Fracaso y abandono escolar en la Educación Secundaria Obligatoria: implicación de las familias y centros escolares, Aula Abierta, 85, pp. 127-146. 
MCCLUSKEY, C., BYNUM, T. y PATCHIN, J. (2004): Reducing chronic absenteeism: An assessment of an early truancy initiative, Crime \& Delinquency, 50, pp. 214-234.

MINISTERIO DE EDUCACIÓN (2009): Panorama de la educación. Indicadores de la OCDE 2009 Informe español (Madrid, Instituto de Evaluación, Dirección General de Evaluación y Cooperación Territorial, Secretaría de Estado de Educación y Formación Profesional).

NAVARRETE (2007): Jóvenes y fracaso escolar en España (Madrid, Fundación INJUVE) (http://www.injuve.migualdad.es/injuve/contenidos. item. action id $=893571637 \&$ menuId=686639162), consultado el 6 noviembre de 2010.

NEWSOME, W. (2004): Solution-Focused Brief Therapy Groupwork with At-Risk Junior High School Students: Enhancing the Bottom Line, Research on Social Work Practice, 14/5, pp. 336-343.

RISSO, A., PERALBO, M. y BARCA, A. (2010): Cambios en las variables predictoras del rendimiento escolar en Enseñanza Secundaria, Psicothema, 22/4, pp. 790-796.

RUÉ, J. (2005): El absentismo escolar como reto para la calidad educativa (Madrid, Ministerio de educación y ciencia \& Subdirección General de Información y Comunicaciones).

SÁEZ, L. (2005): La educación social: intervención socioeducativa en la problemática del absentismo escolar, INDIVISA, Boletín de Estudios e Investigación, 6, pp. 229-240.

WITMER, J. (2007): Youth at Risk for Truancy Detour into a Faith-Based Education Program: Their Perceptions of the Program and its Impact, Research on Social Work Practice, 17/2, pp. 246-257.

\section{PROFESIOGRAFÍA}

\section{$M^{a}$ Isabel Miñaca}

Diplomada en Educación Social y Licenciada en Psicología. Máster de Piscología de la Intervención Social. Técnico Investigador en el Proyecto I+D+i SEJ2007-68099/EDU: «Programa de Tutorías Entre Compañeros (PTEC) para prevenir problemas de rendimiento académico y abandono de los estudios universitarios», así como Investigadora Colaboradora en el Proyecto de 
Innovación Docente 10-53: «ACTÚA: Programa autaplicable dirigido a mejorar la autorregulación del aprendizaje del alumnado universitario de nuevo ingreso e incrementar su rendimiento académico, y en el Proyecto «Hermano Mayor Maracena Educa», con el grupo de investigación HUM 232, del Departamento de Psicología Evolutiva y de la Educación de la Universidad de Granada. Actualmente impartiendo docencia en la Universidad de Huelva.

Datos de contacto: Calle Pascual Martínez, 4, $2^{\circ}$ C. 21006, Huelva (España). E-mail: maribelml@ugr.es

\section{Mirian Hervás}

Licenciada en Pedagogía, Diploma de Estudios Avanzados en el Doctorado Fundamentos del Currículm y Formación del Profesorado en Primaria y Secundaria, en la Universidad de Granada (UGR). Estancia de Posgrado en la Universidad Nacional Autónoma de México. Colaboradora en programa Violencia Escolar para la asociación Daphne, becaria de apoyo técnico a la innovación y mejora docente de la Universidad de Granada, así como investigadora colaboradora en el proyecto «Hermano Mayor - Maracena Educa-, con el grupo de investigación HUM 232, del Departamento de Psicología Evolutiva y de la Educación de la UGR. Becaria con cargo al Servicio General y Apoyo Técnico, con destino en la Oficina de Relaciones Internacionales en la Facultad de Ciencias. Actualmente impartiendo docencia en la Univerisdad de Huelva.

Datos de contacto: Calle Ronda del Alfarero, 5, $1^{\circ}$ III. 18011, Granada (España).E-mail: miriamhervas@ugr.es

Fecha de recepción: 21 de febrero de 2012

Fecha de revisión: 27 de junio de 2012 y 29 de junio de 2012

Fecha de aceptación: 9 de julio de 2012 